\title{
VIDEOTORACOSCOPIA NO TRAUMA DE TÓRAX
}

\author{
VIDEOTHORACOSCOPY IN THORACIC TRAUMA
}

\author{
Vicente Dorgan Neto, TCBC-SP ${ }^{1}$ \\ Roberto Saad Júnior, TCBC-SP ${ }^{2}$ \\ Samir Rasslan, TCBC-SP 3
}

\begin{abstract}
RESUMO: Objetivos: Com o intuito de definir a função da videotoracoscopia no diagnóstico e tratamento no trauma torácico, foram estudados 51 traumatizados por traumas penetrantes ocasionados por arma branca, ferimentos por projétil de arma de fogo ou traumas fechados do tórax, com suspeita diagnóstica por exame clínico e/ ou radiológico de lesões torácicas. Métodos: Foram selecionados doentes estáveis vítimas de trauma torácico (pressão arterial sistólica igual ou superior a 90mmHg) com diagnóstico de: hemotórax em 20 (cinco hemotórax estacionários, quatro hemotórax progressivos e 11 hemotórax coagulados), contusões e ferimentos precordiais (três), ferimentos da zona de transição tóraco-abdominal (24), corpo estranho no tórax (dois) e ferimentos transfixantes do mediastino (dois). Todos foram submetidos à videotoracoscopia. Resultados: A videotoracoscopia se mostrou eficiente na investigação diagnóstica nos casos de hemotórax progressivo (quatro casos), hemotórax coagulado (11 casos), contusões e ferimentos precordiais (três casos), ferimentos da zona da transição tóracoabdominal (confirmação de nove lesões diafragmáticas em 24 traumatizados examinados, 37,5\%) e corpos estranhos no tórax, retirada do corpo estranho com sucesso (dois casos). O procedimento também foi eficiente, além do diagnóstico, no tratamento de hemotórax progressivo (ligadura de artéria mamária um caso, cauterização de vasos intercostais um caso), hemotórax coagulado (remoção de coágulos e decorticação, 11 casos) tendo evitado a realização de toracotomia em 33,3\% dos traumatizados examinados. Conclusões: A videotoracoscopia é método eficiente para diagnóstico e tratamento no traumatismo do tórax e ainda pode evitar a toracotomia em expressivo número de pacientes submetidos ao procedimento.
\end{abstract}

Descritores: Traumatismos do tórax, Cirurgia endoscópica e Videotoracoscopia.

\section{INTRODUÇÃO}

Na última década, com a introdução da câmera de vídeo acoplada ao sistema óptico, a videotoracoscopia começou a ser freqüentemente utilizada em todo o mundo para diagnóstico e tratamento de afecções torácicas no trauma. As primeiras referências surgiram a partir de 1992, considerando a videotoracoscopia como interessante recurso, a ser avaliado, para diagnóstico e trata- mento nas diversas situações de emergência frente ao trauma torácico ${ }^{1-8}$.

A toracoscopia convencional é conhecida desde o início deste século ${ }^{9}$, no entanto existem poucas referências à toracoscopia convencional para o diagnóstico e tratamento do trauma torácico. A primeira delas, todavia, é atribuída a Branco ${ }^{10}$, que realizou o esvaziamento de hemotórax traumático em cinco casos. Outros autores usaram a toracoscopia para diagnóstico de lesão diafragmática, tratamento de

1. Professor Assistente, Doutor do Departamento de Cirurgia, Disciplina de Cirurgia Torácica.

2. Professor Titular da Disciplina de Cirurgia Torácica e Chefe da Disciplina de Cirurgia Torácica.

3. Professor Titular da Disciplina de Cirurgia de Emergência e Diretor do Serviço de Emergência.

Recebido em 18/5/1999

Aceito para publicação em31/10/2000

Trabalho realizado no Serviço de Emergência do Departamento de Cirurgia da Faculdade de Ciências Médicas da Santa Casa de São Paulo, com apoio do CNPq - Conselho Nacional de Desenvolvimento Científico e Tecnológico. 
lesões de artérias intercostais e esvaziamento de hemotórax coagulado; já na década de 1980 os autores consideraram a toracoscopia (convencional) como método de escolha para algumas situações no trauma de tórax ${ }^{11-13}$.

Contudo, a toracoscopia convencional no trauma era utilizada em alguns poucos serviços na Europa e América do $\mathrm{Sul}^{13}$.

A experiência mostra que apenas $10 \%$ a $15 \%$ dos traumatizados do tórax necessitam toracotomia ${ }^{14}$, e os demais $85 \%$ têm resolução com procedimentos menos invasivos, na maioria das vezes com a drenagem fechada do tórax; no entanto, com estes procedimentos simples não é possível visualizar o diafragma e outras estruturas intrapleurais podendo algumas lesões ficar sem diagnóstico. A intervenção tardia nestas situações, quando as manifestações clínicas ou exames complementares a indicam, tem habitualmente como consequiência a realização de operações de grande porte, com maior trauma cirúrgico, maior possibilidade de infecções e frequientemente morbidade mais elevada.

Levando em consideração os aspectos acima relatados e o entusiasmo com a videotoracoscopia, interessamonos pela realização deste procedimento em vítimas de trauma do tórax, com o objetivo de definir sua função no diagnóstico e tratamento especificamente em casos de hemotórax, contusões e ferimentos precordiais, ferimentos da zona da transição tóraco-abdominal, corpos estranhos no tórax e ferimentos transfixantes do mediastino.

\section{MÉTODOS}

Para a seleção de vítimas de traumatismo do tórax a serem submetidas à videotoracoscopia, tanto para diagnóstico como para tratamento, alguns critérios foram estabelecidos após atendimento inicial no serviço de emergência, a saber:

A. Estabilidade hemodinâmica, com pressão arterial sistólica igual ou superior a $90 \mathrm{mmHg} \mathrm{e}$

B. Frequiência respiratória entre 14 e 20 movimentos por minuto.

Foram estabelecidos como condições para a indicação de videotoracoscopia os traumas em que o exame clínico e/ou radiológico do tórax revelaram:

1. Hemotórax.

2. Contusões e ferimentos precordiais.

3. Ferimentos da zona da transição tóraco-abdominal.

4. Corpo estranho no tórax.

5. Ferimentos transfixantes do mediastino.

De acordo com os aspectos clínicos, foram considerados os seguintes tipos de hemotórax: Hemotórax estacionário, caracterizado por presença de sangue na cavidade pleural com volume que variou entre $300 \mathrm{ml}$ e $1.500 \mathrm{ml}$ e volume de drenagem inferior a $150 \mathrm{ml} / \mathrm{h}$ durante 24 horas; hemotórax progressivo, caracterizado por sangramento persistente pelo dreno de tórax $(150 \mathrm{ml} / \mathrm{h}$ a $200 \mathrm{ml} / \mathrm{h})$ ou por significante aumento da imagem de opacificação verificado no exame radiológico do tórax de controle; e hemotórax coagulado, caracterizado pela presença de hemotórax sem expansão pulmonar desde três dias até 90 dias após o trauma.
No período de março de 1993 até maio de 1998, 51 traumatizados de tórax, sendo 44 do sexo masculino e sete do feminino, com idade de 15 a 89 anos (mediana de 31 anos), foram submetidos à videotoracoscopia no Serviço de Emergência do Departamento de Cirurgia da Santa Casa de São Paulo.

Estes traumatizados foram atendidos com traumas penetrantes ocasionados por arma branca em 27 doentes, ferimentos por projétil de arma de fogo em 15 ou traumas fechados de tórax em nove.

Após anestesia geral com intubação seletiva ou não, os traumatizados foram posicionados em decúbito lateral, com o lado comprometido para cima, posição semelhante à utilizada nas toracotomias convencionais.

Com a sonda de intubação de duplo lúmen, a ventilação para o pulmão ipsilateral era bloqueada antes do primeiro acesso à cavidade pleural; quando utilizada a sonda de único lúmen, esta era desconectada do sistema de ventilação durante o primeiro acesso à cavidade pleural.

Em alguns traumatizados, foi utilizado o próprio orifício de penetração de arma branca para inspeção da cavidade pleural com o sistema óptico ou para colocação de instrumentos endoscópicos e convencionais para apresentação das estruturas ou realização de procedimentos.

Em alguns casos, foram necessárias incisões auxiliares ou ampliação do próprio ferimento penetrante com extensão de três a dez centímetros, para o tratamento das lesões intrapleurais.

Tanto a sonda de intubação seletiva como a de intubação de único lúmen foram adequadas para a realização da videotoracoscopia.

A sonda de intubação de duplo lúmen foi utilizada em 30 traumatizados $(58,82 \%)$ e a de único lúmen, em $21(41,18 \%)$.

\section{RESULTADOS}

Dos 20 traumatizados com hemotórax, cinco apresentavam hemotórax estacionário; quatro, progressivo e 11, coagulado.

No hemotórax estacionário, em três traumatizados existia apenas sangue intrapleural, e, em dois, lesão de parênquima pulmonar sem sangramento ativo. Todos estes traumatizados foram tratados com aspiração, lavagem da cavidade pleural com $3.000 \mathrm{ml}$ de soro fisiológico e drenagem fechada do tórax.

Em um destes cinco traumatizados havia hematoma intraparenquimatoso; na evolução apresentou broncopneumonia e teve alta após nove dias de internação.

Dos quatro traumatizados submetidos à videotoracoscopia com hemotórax progressivo, em dois foi necessária a realização de toracotomia, por existirem lesões extensas do parênquima pulmonar e sangramento ativo de vasos pulmonares de maior calibre, que foram suturados após ampliação de uma das incisões da videotoracoscopia. Realizou-se ligadura de artéria mamária em um doente e cauterização de vasos intercostais em outro, com pequena incisão de três centímetros de extensão (visualização pelo sistema óptico) próxima ao local de sangramento. 
Em 11 traumatizados com hemotórax coagulado, nove tiveram evolução satisfatória com expansão pulmonar após a videotoracoscopia. Durante o procedimento, foi feita a remoção de coágulos, decorticação pulmonar, aspiração, lavagem da cavidade pleural com $3.000 \mathrm{ml}$ de soro fisiológico e drenagem fechada do tórax. Estes doentes receberam alta hospitalar, em média, após cinco dias de internação.

Em um traumatizado com elevação de cúpula frênica após 30 dias do trauma, foi indicada a videotoracoscopia e realizada decorticação, lavagem e drenagem do tórax. Após uma semana do procedimento, não tendo ocorrido expansão satisfatória do pulmão, foi submetido à toracotomia, tendo sido identificada lesão de $15 \mathrm{~cm}$ de extensão no diafragma e lesão do fígado.

Em um dos pacientes submetidos à videotoracoscopia e à decorticação pulmonar, apesar de ter ocorrido expansão pulmonar após o procedimento, durante a intubação com sonda de único lúmen houve lesão de traquéia membranosa. Na evolução pós-operatória, apresentou enfisema do mediastino e subcutâneo, falência de múltiplos órgãos e morte no 11을 dia de pós-operatório.

Foram submetidos à videotoracoscopia três pacientes com contusões e ferimentos precordiais. Em um portador de trauma fechado, observou-se pela videotoracoscopia apenas hemopericárdio; nos outros dois traumatizados, observou-se hemopericárdio e lesão em ventrículo esquerdo, que foram tratados com sutura após toracotomia anterior esquerda. Em um deles, vítima de ferimento por projétil de arma de fogo, havia lesão de ventrículo esquerdo e diafragma, e o orifício de entrada situava-se em linha hemiclavicular, 3 o espaço intercostal esquerdo. No outro, com ferimento por arma branca, havia lesão de ventrículo esquerdo, e o orifício de entrada encontrava-se em linha hemiclavicular, 5o espaço intercostal esquerdo

Vinte e quatro traumatizados com trauma penetrante abaixo do $4^{\circ}$ espaço intercostal, na zona de transição tóraco-abdominal (orifício de entrada ou saída), foram submetidos à videotoracoscopia para diagnóstico de lesão diafragmática, que foi confirmada em nove deles $(37,5 \%)$.

Em três (12,5\%), apesar da integridade do diafragma, observou-se sangramento ativo em vasos da parede torácica, que foram tratados com cauterização pela videotoracoscopia. Em seis traumatizados (25\%), observou-se hemotórax e lesão em parênquima pulmonar, sem sangramento ativo durante o procedimento; foram tratados com aspiração, lavagem da cavidade pleural com $3.000 \mathrm{ml}$ de soro fisiológico e drenagem fechada do tórax. Em um caso $(4,17 \%)$, foi necessária toracotomia e sutura do pulmão devida à lesão extensa no parênquima pulmonar, sangramento ativo e escape de ar. Em três outros (12,5\%), observou-se apenas hemotórax; foram tratados com aspiração, lavagem da cavidade pleural com $3.000 \mathrm{ml}$ de soro fisiológico e drenagem fechada do tórax. Por fim, em um destes pacientes $(4,17 \%)$ não existia hemotórax nem qualquer lesão, exceto pelo orifício de penetração de projétil de arma de fogo no tórax; foi tratado com drenagem fechada do tórax.

Dos pacientes com corpo estranho no tórax foram tratados dois traumatizados com arma branca (faca) encra- vada no precórdio. Durante a videotoracoscopia, observouse lesão em lobo superior esquerdo sem sangramento e hemotórax de 300ml em um deles e, no outro, lesão em lobo inferior do pulmão esquerdo, também sem sangramento, e hemotórax de $300 \mathrm{ml}$. Foram tratados com retirada da arma branca, com visualização pela videotoracoscopia, aspiração e lavagem da cavidade pleural com $3.000 \mathrm{ml}$ de soro fisiológico e drenagem fechada do tórax.

A presença de ferimentos transfixantes do mediastino resultou na indicação de videotoracoscopia em dois traumatizados. Um era vítima de ferimento por projétil de arma de fogo, com orifício de entrada junto à fúrcula esternal e saída em 5o espaço intercostal esquerdo, próximo da ponta da escápula, e foi submetido à arteriografia que não mostrou qualquer lesão. Durante a videotoracoscopia observou-se apenas hemotórax de $700 \mathrm{ml}$ que foi tratado com aspiração, lavagem da cavidade pleural com $3.000 \mathrm{ml}$ de soro físiológico e drenagem fechada do tórax.

O outro era vítima de ferimentos por arma branca em 5 o espaço intercostal à direita e à esquerda na região paravertebral. No exame de entrada ao Serviço de Emergência apresentava-se estável e com enfisema subcutâneo de grande volume. Durante a videotoracoscopia direita observaram-se bolhas pulmonares (preexistentes), pneumomediastino e múltiplas lesões em pulmão direito; durante a videotoracoscopia à esquerda observaram-se também bolhas pulmonares e lesão em brônquio fonte esquerdo e parênquima próximo ao hilo do pulmão esquerdo. Neste caso foi indicada esternotomia longitudinal para tratamento das múltiplas lesões bilaterais.

Em 50 traumatizados a videotoracoscopia foi eficiente para identificação ou exclusão de lesões decorrentes de trauma penetrante e trauma fechado de tórax $(98,04 \%)$, sendo que em 17 deles foram realizados procedimentos terapêuticos $(33,33 \%)$. Em um, a videotoracoscopia não foi eficiente no diagnóstico (Figura 1), pois existiam lesão diafragmática extensa e lesão hepática que foram confirmadas somente após realização da toracotomia (Tabelas 1 e 2).

Tabela 1

Distribuição dos traumatizados de acordo com os diagnósticos confirmados após realização da videotoracoscopia

\begin{tabular}{l|c}
\hline Diagnósticos & No de traumatizados \\
\hline Hemotórax & 50 \\
Lesão de diafragma & 10 \\
Lesão pulmonar & 10 \\
Lesão de vasos parietais & 5 \\
Lesão pulmonar extensa & 4 \\
Lesão de pericárdio & 3 \\
Lesão cardíaca & 2 \\
Lesão de brônquio fonte esquerdo & 1 \\
Ausência de lesões & 1 \\
\hline
\end{tabular}


Tabela 2

Distribuição dos traumatizados de acordo com os procedimentos terapêuticos realizados com utilização da videotoracoscopia

\begin{tabular}{l|c|l}
\hline Diagnósticos & No de Traumatizados & Procedimentos Terapêuticos \\
\hline Encarceramento pulmonar & 10 & Decorticação pulmonar \\
Lesão de vasos parietais & 4 & Cauterização de vasos intercostais \\
$\begin{array}{l}\text { Retirada de corpo } \\
\text { estranho no tórax }\end{array}$ & 2 & $\begin{array}{l}\text { Retirada de arma branca com visualização pela } \\
\text { videotoracoscopia }\end{array}$ \\
$\begin{array}{l}\text { Lesão de artéria mamária } \\
\text { Total }\end{array}$ & 1 & Incisão auxiliar e ligadura \\
\hline
\end{tabular}

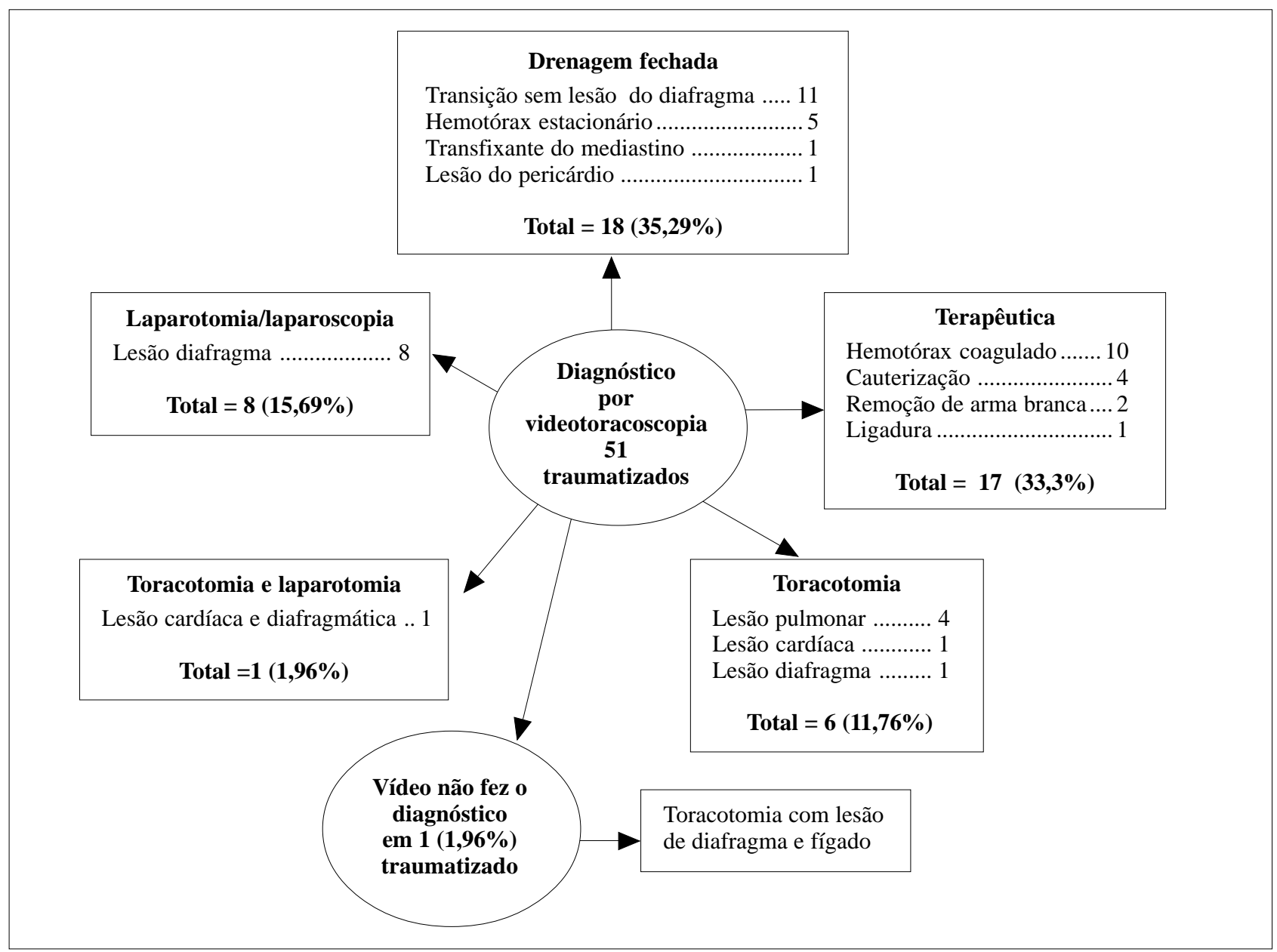

Figura 1. Tratamento definitivo após realização da videotoracoscopia em 51 traumatizados com trauma de tórax.

Não houve complicações que pudessem ser atribuídas ao procedimento videotoracoscópico. O tempo cirúrgico foi em média de uma hora. Os traumatizados tiveram alta em média cinco dias após os procedimentos. O que teve alta mais precoce permaneceu um dia internado; o que teve internação pro- longada permaneceu 30 dias no hospital após toracotomia e tratamento de extensa lesão de parênquima pulmonar.

Ocorreram três óbitos $(5,8 \%)$ que não foram atribuídos ao procedimento videoendoscópico, mas sim à gravidade das lesões ou a doenças preexistentes. 


\section{DISCUSSÃo}

Nos serviços de emergência o uso da toracoscopia ainda tem sido eventual,pois os cirurgiões da emergência não estão habituados com a técnica do procedimento que, apesar de não ser complexa, requer treinamento especial.

A videotoracoscopia no tratamento do hemotórax estacionário, apesar de permitir a identificação da causa exata do sangramento intrapleural e facilitar o esvaziamento do hemotórax de maneira rápida e eficiente, é realizada com anestesia geral, o que representa maior agressão cirúrgica quando comparada à drenagem fechada do tórax, que habitualmente é praticada com anestesia local e pequeno trauma cirúrgico. Acreditamos que a drenagem fechada deve ser o tratamento de escolha no hemotórax estacionário, pois é eficiente, de execução simples e com mínima agressão anestésico-cirúrgica.

No hemotórax progressivo ou contínuo, a videotoracoscopia permite definir a causa do sangramento e efetuar o tratamento através de incisões que propiciam menor agressão cirúrgica se comparadas com a toracotomia convencional $^{15-19}$. Mesmo com a toracoscopia convencional outros autores anteriormente chegaram a tais conclusões ${ }^{12}$.

O hemotórax coagulado ou tardio geralmente é conseqüência do insucesso no tratamento habitual por drenagem fechada que pode ser atribuído à falha técnica na execução da drenagem, presença de lesões não diagnosticadas ou então à gravidade do trauma caracterizada por extensas lesões intrapleurais. O acompanhamento clínico e radiológico deve fornecer subsídios para a indicação da drenagem e esta deve ser a mais precoce possível, evitando desta maneira a ocorrência de empiema pleural, encarceramento pulmonar e suas conseqüências.

Dos nossos traumatizados, quatro vieram de outros serviços, e, apesar de terem sido encaminhados entre dez e 20 dias após o tratamento inicial, tiveram boa resolução após a videotoracoscopia. Em apenas um caso, o método videoendoscópico não foi eficiente para o diagnóstico e expansão pulmonar. Tratava-se de doente com trauma fechado em base do hemitórax direito há 30 dias sem expansão do pulmão, e a videotoracoscopia não permitiu a identificação de grande lesão diafragmática com todo o fígado ocupando o limite inferior da cavidade pleural; somente com a toracotomia convencional e após exame minucioso da base do hemitórax direito foi possível identificar o parênquima hepático intrapleural, visto que a deposição de fibrina e o processo inflamatório local dificultaram a avaliação pela videotoracoscopia.

Nos traumas da região precordial em doentes estáveis, a janela pericárdica subxifóide é procedimento eficiente e consagrado que permite definir com alta sensibilidade, especificidade e acurácia se existe ou não sangue no saco pericárdico ${ }^{20}$; porém, não avalia a existência de outras lesões intratorácicas. A videotoracoscopia realizada em número expressivo de traumatizados ${ }^{21}$ mostrou tratar-se de método recomendado para diagnóstico em traumatizados com suspeita de lesão cardíaca.

Nos três traumatizados com indicação de janela pericárdica, a videotoracoscopia mostrou, em dois doentes, lesão de miocárdio e, em um, lesão do pericárdio. Apesar do pequeno número de casos com confirmação de lesão cardíaca, a utilização da videotoracoscopia nesta situação permitiu diagnosticar outras lesões intratorácicas que não teriam sido diagnosticadas por janela pericárdica convencional, pois, em um destes traumatizados com lesão de ponta do ventrículo esquerdo, existia também lesão do diafragma que foi tratada após a sutura da lesão cardíaca através de incisão auxiliar em 6o espaço intercostal esquerdo anterior de dez centímetros de extensão.

Acreditamos que a videotoracoscopia com objetivo de diagnosticar lesões cardíacas no trauma é eficiente, não representa maior agressão cirúrgica se comparada à janela pericárdica subxifóide e, ainda, possibilita a visualização da cavidade pleural com a finalidade de diagnóstico e tratamento de outras lesões intrapleurais. Nos ferimentos precordiais, abaixo do 4o espaço intercostal, parede anterior do tórax, em doentes estáveis, a videotoracoscopia deve ser o método de escolha, pois permite, além de investigar a lesão cardíaca, examinar a zona da transição tóraco-abdominal.

Nos ferimentos da zona da transição tóraco-abdominal, foi utilizada em 24 traumatizados, e se mostrou adequada para a confirmação, exclusão e/ou realização de outros diagnósticos ${ }^{2,3,22,23} \mathrm{e}$, eventualmente, realização do tratamento definitivo em traumatizados selecionados, pois tanto no trauma fechado como nos ferimentos penetrantes o diagnóstico pelos métodos de imagem não é eficiente em pelo menos $30 \%$ dos $\operatorname{casos}^{24}$. Confirmamos lesão diafragmática em nove destes $(37,5 \%)$. Se tivéssemos utilizado a via abdominal para confirmação de lesão diafragmática nestes 24 casos, teríamos violado a cavidade abdominal sem lesões preexistentes em 15 deles (62,5\%), e em alguns destes traumatizados foi diagnosticada lesão pulmonar extensa e sangramento ativo de vasos intercostais que necessitaram tratamento pela videotoracoscopia ou toracotomia.

No corpo estranho encravado no tórax, pela possibilidade de estar tamponando eventual lesão de grande vaso intratorácico ou coração, sua remoção deve ser feita com visão direta do seu trajeto pela toracotomia ou videotoracoscopia. Quando se utiliza a videotoracoscopia, se não existir tamponamento de grandes vasos ou coração, a retirada do corpo estranho pode ser feita sem toracotomia e com segurança.

Tivemos oportunidade de realizar o procedimento videotoracoscópico em duas lesões mediastinais, em uma permitiu definir a via de acesso para toracotomia e em outra foi constatado hemotórax. É necessário um maior número de traumatizados para que se possam definir as vantagens do seu emprego. A avaliação desta pequena casuística mostra que a videotoracoscopia no trauma torácico surge como importante recurso para diagnóstico de lesões cardíacas e diafragmáticas em doentes assintomáticos, diagnóstico e tratamento no hemotórax progressivo, hemotórax coagulado e remoção de corpos estranhos. Deve ser indicada sempre que possível pois é procedimento eficiente e seguro e evita a realização de toracotomia em número expressivo de doentes.

Em situações como o quilotórax traumático ${ }^{2,19}$ e ferimentos transfixantes do mediastino a definição das vantagens da utilização da videotoracoscopia, no diagnóstico e tratamento, merece ainda um estudo. 


\begin{abstract}
Background: The videothoracoscopy in diagnosis and management of thoracic trauma was evaluated, 51 individuals attended in the Emergency Service of the Surgery Department of Santa Casa de São Paulo with the intention of defining the function of the procedure in cases of penetrating trauma caused by stab, fire-arms projectile or blunt, in diagnosis suspected clinical or radiologic of thoracic lesions. Methods: Were selected patients victims of thoracic trauma with diagnosis of hemothorax, precordial contusions and wounds, wounds of thoracic-abdominal transition, embedded knife in the chest and transfixing wounds of mediastinum. The procedures were accomplished in having traumatized stable (blood pressure same or superior $90 \mathrm{mmHg}$ ). All of them were submitted to videothoracoscopy. Results: videoassisted thoracic surgery is an efficient procedure in the diagnostic investigation, in cases of progressive hemothorax (4 cases) and coagulated hemothorax (11 cases), precordial contusions and wounds (3 cases), wounds of thoracic-abdominal transition (24 cases, diaphragmatic injury was confirmed in 9, 37.5\%) and embedded knife from the chest (2 cases). The procedure was efficient as well in the management of progressive and coagulated hemothorax and to remove embedded knife from the chest, having avoided the thoracotomy in 33.3\% of the examined individuals. Conclusions: the videothoracoscopy is efficient procedure for diagnosis and treatment in thoracic trauma and it can still avoid the thoracotomy in expressive number of patients submitted to the procedure
\end{abstract}

Key Words: Thoracic trauma; Endoscopic surgery and Videoassisted thoracic surgery.

\title{
REFERÊNCIAS
}

1. Feliciano DV. The diagnostic and therapeutic approach to chest trauma Semin. Thorac Cardiovasc Surg, 1992. 4:156-162.

2. Kern JA, Tribble CG, Spotnitz WD et al. Thoracoscopy in subacute management of pacients with thoracoabdominal trauma. Chest, 1993. 104:942-945.

3. Roviaro GC, Rebuffat C, Varolif et al. Videoendoscopic thoracic surgery. Int Surg, 1993. 78:4-9.

4. Saad Júnior R, Rasslan S. A videocirurgia no trauma de tórax. [Editorial]. Rev Col Bras Cir 1993. 20.

5. Saad Júnior R, Rasslan S. Complicações da videocirurgia torácica. In: Coelho JC, Marchesin JB, Malafaia O. Complicações da vídeo-cirurgia, Rio de Janeiro, Medsi, 1994. p. 413-26.

6. Saad Júnior R, Dorgan Neto V, Aguiar Neto J R. Análise crítica do tratamento videocirúrgico para os traumatismos torácicos. In: Margarido N F, Saad Júnior R, Cecconello I, Martins JL, De Paula RA, Soares LA. Vídeo Cirurgia, São Paulo, Robe Editorial, 1994. p.317-23.

7. Rasslan S, Rodrigues FCM, Soldá SC et al. Corpo estranho intratorácico tratado por videocirurgia. Rev Col Bras Cir, 1994. 21:42-44.

8. Forte V. O papel da cirurgia torácica vídeo-assistida (CTVA). [Editorial]. J Pneumol, 1994. 20:1.

9. Jacobaeus HC. Uber die moglichkeit die zystokopie bei untersuchung seroser hohlungen anzuwenden. Med Wochenschr, 1910. 57:2090-2092.

10. Branco JMJ. Thoracoscopy as a method of exploration in penetrating injuries of the thorax. Dis Chest 1946. 12:330-335.

11. Jackson AM, Ferreira AA. Thoracoscopy as an aid to the diagnosis of diaphragmatic injury in penetrating wounds of the lower chest.: a preliminary report. Injury, 1976. 14:213-217.

12. Jones JW, Kitahama A, Webb WR et al. Emergency thoracoscopy: a logical approach to chest trauma management. J Trauma, 1981. 21:280-284.

13. Adamthwaite DN. Penetrating injuries of the diaphragm. Injury, 1982. 14:151-158.

14. Saad Júnior R, Dorgan Neto V, Aguiar Neto J R et al. Traumatismos torácicos : Sistematização na avaliação ini- cial. Arq. Med. Hosp. Fac. Ciên. Med. Santa Casa São Paulo, 1989. 9:70-72.

15. Potapenkov MA, Shipulin PP, Prokhoda SA. Emergency thoracoscopy in the diagnosis and treatment of the complicated chest injury. Grud. Serdechnososudistaia Khir., 1992. 5:32-35.

16. Smith RS, Fry WR, Tsoi EK et al. Preliminary report on videothoracoscopy in the evaluation and treatment of thoracic injury. Am J Surg, 1993. 166:690-693.

17. Liu HP, Chang $\mathrm{CH}$, Lin PJ et al. Video-assisted thoracic surgery. The Chang Gung experience. J Thorac Cardiovasc Surg, 1994. 108:834-840.

18. Wong MS, Tsoi EK, Henderson VJ et al. Videothoracoscopy an effective method for evaluating and managing thoracic trauma patients. Surg Endosc, 1996. 10:118-121.

19. Lang-Lazdunski L, Mouroux J, Pons F et al. Role of videothoracoscopy in chest trauma. Ann. Thorac. Surg., 1997. 63:327-333.

20. Prado PA, Saad Júnior R, Rasslan S. O emprego da janela pericárdica no diagnóstico do ferimento cardíaco. Rev Col Bras Cir, 1995. 22:307-312.

21. Morales CH, Salinas CM, Henao CA et al. Thoracoscopic pericardial window and penetrating cardiac trauma. J Trauma, 1997. 42:273-275.

22. Ochsner MG, Rozycki GS, Lucente F et al. Prospective evaluation of thoracoscopy for diagnosing diaphagmatic injury in thoracoabdominal trauma: a preliminary report. J Trauma, 1993. 34: 709-710.

23. Uribe RA, Pachon CE, Frame SB et al. A prospective evaluation of thoracoscopy for the diagnosis of penetrating thoracoabdominal trauma. J Trauma, 1994. 37:650-654.

24. Madden MR, Paull DE, Finkelstein JL et al. Occult diaphragmatic injury from stab wounds to the lower chest and abdomen. J Trauma, 1989. 29:292-298.

Endereço para correspondência:

Dr. Vicente Dorgan Neto

Av. Itacira, 965

04061-002 - São Paulo-SP 